Bull. Korean Math. Soc. 49 (2012), No. 6, pp. 1291-1302

http://dx.doi.org/10.4134/BKMS.2012.49.6.1291

\title{
A NEW ALTERNATIVE ELLIPTIC PDE IN EIT IMAGING
}

\author{
SungWhan Kim
}

ABstract. In this paper, we introduce a new elliptic PDE:

$$
\left\{\begin{array}{cc}
\nabla \cdot\left(\frac{\left|\gamma^{\omega}(\mathbf{r})\right|^{2}}{\sigma} \nabla v_{\omega}(\mathbf{r})\right)=0, & \mathbf{r} \in \Omega, \\
v_{\omega}(\mathbf{r})=f(\mathbf{r}), & \mathbf{r} \in \partial \Omega,
\end{array}\right.
$$

where $\gamma^{\omega}=\sigma+i \omega \epsilon$ is the admittivity distribution of the conducting material $\Omega$ and it is shown that the introduced elliptic PDE can replace the standard elliptic PDE with conductivity coefficient in EIT imaging. Indeed, letting $v_{0}$ be the solution to the standard elliptic PDE with conductivity coefficient, the solution $v_{\omega}$ is quite close to the solution $v_{0}$ and can show spectroscopic properties of the conducting object $\Omega$ unlike $v_{0}$. In particular, the potential $v_{\omega}$ can be used in detecting a thin low-conducting anomaly located in $\Omega$ since the spectroscopic change of the Neumann data of $v_{\omega}$ is inversely proportional to thickness of the thin anomaly.

\section{Introduction}

Let $\Omega$ be a two- or three-dimensional conducting bounded object with Lipschitz boundary $\partial \Omega$ and let $D$ be an anomaly located inside $\Omega$. Since the admittivity distribution $\gamma^{\omega}$ of the conducting object $\Omega$ changes abruptly across the anomaly, we suppose that the admittivity distribution $\gamma^{\omega}$ is defined by

$$
\gamma^{\omega}:=\sigma+i \omega \epsilon= \begin{cases}\sigma_{b}+i \omega \epsilon_{b} & \text { in } \Omega \backslash \bar{D} \\ \sigma_{c}+i \omega \epsilon_{c}, & \text { in } D .\end{cases}
$$

In this paper, we want to reconstruct the anomaly $D$ using electrical impedance tomography (EIT) image methods. To do so, through the boundary $\partial \Omega$ we apply a sinusoidal voltage of $f(\mathbf{r}) \sin \omega t$ with its frequency $\frac{\omega}{2 \pi}$, where $f \in$ $H^{1 / 2}(\partial \Omega)$ is a non-zero real valued function. Then the corresponding time harmonic complex potential $u_{\gamma^{\omega}}:=v_{\gamma^{\omega}}+i h_{\gamma^{\omega}}$ satisfies the following Dirichlet

Received May 26, 2011; Revised April 4, 2012.

2010 Mathematics Subject Classification. Primary 35J25, 35A35, 35R30.

Key words and phrases. electrical impedance tomography, alternative elliptic PDE, spectroscopic change, anomaly thickness.

This work was supported by the Korea Research Foundation Grant funded by the Korean Government (KRF-2007-521-C00022). 
boundary value problem:

$$
\left\{\begin{array}{l}
\nabla \cdot\left(\gamma^{\omega}(\mathbf{r}) \nabla u_{\gamma^{\omega}}(\mathbf{r})\right)=0, \quad \mathbf{r} \in \Omega \\
u_{\gamma^{\omega}}(\mathbf{r})=f(\mathbf{r}), \quad \mathbf{r} \in \partial \Omega .
\end{array}\right.
$$

It is well known from the Lax-Milgram Lemma that for $f \in H^{1 / 2}(\partial \Omega)$ there exists a unique complex potential $u_{\gamma \omega} \in H^{1}(\Omega)=\left\{\left.\phi\left|\int_{\Omega}\right| \phi\right|^{2}+|\nabla \phi|^{2} d \mathbf{r}<\infty\right\}$. Then the measured exit current

$$
g_{\gamma^{\omega}}:=\underbrace{\mathbf{n} \cdot\left(-\sigma \nabla v_{\gamma^{\omega}}+\omega \epsilon \nabla h_{\gamma^{\omega}}\right)}_{\text {real part }}+i \underbrace{\mathbf{n} \cdot\left(-\sigma \nabla h_{\gamma^{\omega}}-\omega \epsilon \nabla v_{\gamma^{\omega}}\right)}_{\text {imaginary part }} \quad \text { on } \partial \Omega \text {, }
$$

belongs to the space $H^{-1 / 2}(\partial \Omega)$, where $\mathbf{n}$ is the unit outward normal vector to the boundary $\partial \Omega$. In EIT, we investigate the interrelation between the measurable quantity $g_{\gamma^{\omega}}$ and the admittivity distribution $\gamma^{\omega}[2,3,5,6,11,14$, $20]$.

However, it is not easy to deal with the complex elliptic PDE (2), since we have little knowledge on it. We do not even know whether the fundamental properties such as a maximum principle and a weighted mean value type theorem exist. So many researches in EIT consider the following standard elliptic $\mathrm{PDE}$ instead of $(2)$ under the assumption $\omega \epsilon \approx 0$ :

$$
\begin{cases}\nabla \cdot\left(\sigma \nabla v_{0}(\mathbf{r})\right)=0, & \mathbf{r} \in \Omega \\ v_{0}(\mathbf{r})=f(\mathbf{r}), & \mathbf{r} \in \partial \Omega .\end{cases}
$$

Surely it is much easier to deal with (4) than (2) and (4) is quite useful for conductivity imaging of the interested conducting object $\Omega$. Instead, we should abandon the spectroscopic properties of the conducting object with respect to angular frequency $\omega$. In particular, if $D$ is a thin membrane-like anomaly such as cell membranes in biological tissues and cracks in conducting materials, the behavior of current flow in $D$ has remarkable changes as frequency varies above, and provides useful information on $D$. Recently, [15] considered the inverse problem of determining an air void inside a concrete structure and proposed a new non-destructive evaluation (NDE) technique for estimating the thickness of a thin air void using a frequency differential data of the exit current $g_{\gamma} \omega$ in (3). In [13], the spectroscopic admittivity image of a membrane structure was studied and it was illustrated how injection current penetrates the insulating membrane at a sufficiently high frequency and uncloak an anomaly completely enclosed by the membrane.

In order to investigate the spectroscopic properties of the interested conducting object $\Omega$ and obtain useful information on the anomaly $D$ from them, the real elliptic PDE (4) is unsuitable and the complex elliptic PDE (2) is unmanageable. So we introduce an alternative elliptic PDE to (2) and (4) which is easy to handle:

$$
\begin{cases}\nabla \cdot\left(\frac{\left|\gamma^{\omega}(\mathbf{r})\right|^{2}}{\sigma} \nabla v_{\omega}(\mathbf{r})\right)=0, & \mathbf{r} \in \Omega \\ v_{\omega}(\mathbf{r})=f(\mathbf{r}), & \mathbf{r} \in \partial \Omega\end{cases}
$$


In this paper, we will demonstrate that the new elliptic PDE (5) is helpful to EIT imaging as much as the elliptic PDE (4). When the value of susceptivity $\omega \epsilon$ is relatively quite small for conductivity distribution $\sigma$, it will be shown that the solution $v_{\omega}$ to $(5)$ is close to the real part $v_{\gamma^{\omega}}$ of the complex potential $u_{\gamma^{\omega}}$ as much as the solution $v_{0}$ to (4) and it can explain spectroscopic phenomena of the true potential $v_{\gamma^{\omega}}$. Since the elliptic PDE (4) ignores the effect of the susceptivity $\omega \epsilon$, the potential $v_{0}$ does not show spectroscopic phenomena of the true potential $v_{\gamma^{\omega}}$. Furthermore, we will show that such spectroscopic change in the Neumann data of $v_{\omega}$ on the boundary of the conducting object $\Omega$ is closely related with some geometric features of the anomaly $D$ included in $\Omega$, in particular, its thickness when the anomaly $D$ is a thin almost insulating material. In fact, the spectroscopic change in the Neumann data of $v_{\omega}$ on the boundary is quantitatively the total amount of current energy only on the anomaly $D$. If the anomaly $D$ is a thin almost insulating material, the current energy on $D$ is relatively big compared with the current energy outside $D$ and the magnitude of $\nabla v_{\omega}$ in the anomaly $D$ is overall inverse proportional to the thickness of $D$. Therefore the rate of change of the Neumann data of $v^{\omega}$ with respect to angular frequency $\omega$ is concluded to show information on thickness of the thin anomaly $D$.

\section{Closeness between $v_{\omega}$ and $v_{0}$}

For a while, we suppose that the conductivity $\sigma$ and permittivity $\epsilon$ are positive bounded functions on $\Omega$. That is, the admittivity distribution $\gamma_{\omega}$ need not to be a simple piecewise constant function as defined in (1). Let us denote by $M_{\gamma^{\omega}}$ the maximal contrast between $\sigma$ and $\omega \epsilon$ :

$$
M_{\gamma^{\omega}}:=\sup _{\mathbf{r} \in \Omega}\left\{\frac{\omega \epsilon(\mathbf{r})}{\sigma(\mathbf{r})}\right\} .
$$

Through this paper, we assume that $M_{\gamma^{\omega}}$ is small enough, which means that the value of susceptivity $\omega \epsilon$ is relatively quite small for conductivity distribution $\sigma$ as often occur in practice. In a practical case, for instance, the conductivity and permittivity distributions of biological tissues in the frequency range below a few $\mathrm{kHz}$ satisfy that $M_{\gamma^{\omega}} \leq 10^{-2}$. Let us denote by $v_{\gamma^{\omega}}$ and $h_{\gamma^{\omega}}$, respectively, the real and imaginary part of the complex potential $u_{\gamma^{\omega}}$, that is, $u_{\gamma^{\omega}}=$ $v_{\gamma^{\omega}}+i h_{\gamma^{\omega}}$. Then the complex elliptic PDE (2) can be rewritten into the following coupled system:

$$
\nabla \cdot\left[\left(\begin{array}{cc}
\sigma & -\omega \epsilon \\
\omega \epsilon & \sigma
\end{array}\right)\left(\begin{array}{c}
\nabla v_{\gamma^{\omega}} \\
\nabla h_{\gamma^{\omega}}
\end{array}\right)\right]=\left(\begin{array}{l}
0 \\
0
\end{array}\right) \quad \text { in } \Omega
$$

with the Dirichlet data

$$
\left(\begin{array}{l}
v_{\gamma^{\omega}} \\
h_{\gamma^{\omega}}
\end{array}\right)=\left(\begin{array}{l}
f \\
0
\end{array}\right) \quad \text { on } \partial \Omega
$$


Since the coupled system (6) is hard to handle, ignoring the susceptivity $\omega \epsilon$ decouples (6) into

$$
\nabla \cdot\left[\left(\begin{array}{cc}
\sigma & 0 \\
0 & \sigma
\end{array}\right)\left(\begin{array}{c}
\nabla v_{\gamma^{\omega}} \\
\nabla h_{\gamma^{\omega}}
\end{array}\right)\right] \approx\left(\begin{array}{l}
0 \\
0
\end{array}\right) \quad \text { in } \Omega
$$

with the same boundary condition $(7)$, and so we have

$$
v_{\gamma^{\omega}} \approx v_{0} \quad \text { and } \quad h_{\gamma^{\omega}} \approx 0 .
$$

The following theorem shows the exact closeness between $v_{\gamma^{\omega}}, v_{\omega}$, and $v_{0}$ in the sense of the maximal contrast $M_{\gamma} \omega$.

Theorem 2.1. Three functions $v_{\gamma^{\omega}}, v_{\omega}$, and $v_{0}$ satisfy the following estimates:

$$
\int_{\Omega} \sigma\left|\nabla v_{\gamma^{\omega}}-\nabla v_{0}\right|^{2} d \mathbf{r} \leq \frac{2 M_{\gamma^{\omega}}^{4}}{1-2 M_{\gamma^{\omega}}^{4}} \int_{\Omega} \sigma\left|\nabla v_{0}\right|^{2} d \mathbf{r}
$$

and

$$
\int_{\Omega} \sigma\left|\nabla v_{\omega}-\nabla v_{0}\right|^{2} d \mathbf{r} \leq \frac{2 M_{\gamma^{\omega}}^{4}}{1-2 M_{\gamma^{\omega}}^{4}} \int_{\Omega} \sigma\left|\nabla v_{0}\right|^{2} d \mathbf{r} .
$$

Proof. Due to (2), (4), and Hölder's inequality, since $v_{\gamma^{\omega}}$ and $v_{0}$ have the same Dirichlet boundary condition on $\partial \Omega$, we obtain

$$
\begin{aligned}
\int_{\Omega} \sigma\left|\nabla v_{\gamma \omega}-\nabla v_{0}\right|^{2} d \mathbf{r} & =\int_{\Omega}\left(\omega \epsilon \nabla h_{\gamma^{\omega}}\right) \cdot\left(\nabla v_{\gamma^{\omega}}-\nabla v_{0}\right) d \mathbf{r} \\
& \leq\left[\int_{\Omega} \sigma\left|\nabla h_{\gamma^{\omega}}\right|^{2} d \mathbf{r}\right]^{\frac{1}{2}}\left[\int_{\Omega} \frac{\omega^{2} \epsilon^{2}}{\sigma}\left|\nabla v_{\gamma} \omega-\nabla v_{0}\right|^{2} d \mathbf{r}\right]^{\frac{1}{2}} \\
& \leq M_{\gamma^{\omega}}\left[\int_{\Omega} \sigma\left|\nabla h_{\gamma^{\omega}}\right|^{2} d \mathbf{r}\right]^{\frac{1}{2}}\left[\int_{\Omega} \sigma\left|\nabla v_{\gamma} \omega-\nabla v_{0}\right|^{2} d \mathbf{r}\right]^{\frac{1}{2}} .
\end{aligned}
$$

From the above inequality we have

$$
\int_{\Omega} \sigma\left|\nabla v_{\gamma^{\omega}}-\nabla v_{0}\right|^{2} d \mathbf{r} \leq M_{\gamma^{\omega}}^{2} \int_{\Omega} \sigma\left|\nabla h_{\gamma^{\omega}}\right|^{2} d \mathbf{r} .
$$

Next we will estimate the integral in the right side of (11):

$$
\begin{aligned}
\int_{\Omega} \sigma\left|\nabla h_{\gamma^{\omega}}\right|^{2} d \mathbf{r} & =-\int_{\Omega}\left(\omega \epsilon \nabla v_{\gamma} \omega\right) \cdot \nabla h_{\gamma^{\omega}} d \mathbf{r} \\
& \leq\left[\int_{\Omega} \frac{\omega^{2} \epsilon^{2}}{\sigma}\left|\nabla v_{\gamma} \omega\right|^{2} d \mathbf{r}\right]^{\frac{1}{2}}\left[\int_{\Omega} \sigma\left|\nabla h_{\gamma^{\omega}}\right|^{2} d \mathbf{r}\right]^{\frac{1}{2}},
\end{aligned}
$$

and so

$$
\int_{\Omega} \sigma\left|\nabla h_{\gamma^{\omega}}\right|^{2} d \mathbf{r} \leq M_{\gamma^{\omega}}^{2} \int_{\Omega} \sigma\left|\nabla v_{\gamma^{\omega}}\right|^{2} d \mathbf{r}
$$

Combining (11) and (12), we obtain the following estimate

$$
\int_{\Omega} \sigma\left|\nabla v_{\gamma^{\omega}}-\nabla v_{0}\right|^{2} d \mathbf{r} \leq M_{\gamma^{\omega}}^{4} \int_{\Omega} \sigma\left|\nabla v_{\gamma^{\omega}}\right|^{2} d \mathbf{r} .
$$


Finally, since

$$
\int_{\Omega} \sigma\left|\nabla v_{\gamma} \omega\right|^{2} d \mathbf{r} \leq 2 \int_{\Omega} \sigma\left|\nabla v_{\gamma} \omega-v_{0}\right|^{2} d \mathbf{r}+2 \int_{\Omega} \sigma\left|\nabla v_{0}\right|^{2} d \mathbf{r},
$$

we obtain the first estimate:

$$
\int_{\Omega} \sigma\left|\nabla v_{\gamma^{\omega}}-\nabla v_{0}\right|^{2} d \mathbf{r} \leq \frac{2 M_{\gamma^{\omega}}^{4}}{1-2 M_{\gamma^{\omega}}^{4}} \int_{\Omega} \sigma\left|\nabla v_{0}\right|^{2} d \mathbf{r} .
$$

The second estimate can be obtained in similar way:

$$
\begin{aligned}
\int_{\Omega} \sigma\left|\nabla v_{\omega}-\nabla v_{0}\right|^{2} d \mathbf{r} & =-\int_{\Omega}\left(\frac{\omega^{2} \epsilon^{2}}{\sigma} \nabla v_{\omega}\right) \cdot\left(\nabla v_{\omega}-\nabla v_{0}\right) d \mathbf{r} \\
& \leq M_{\gamma^{\omega}}^{2}\left[\int_{\Omega} \sigma\left|\nabla v_{\omega}\right|^{2} d \mathbf{r}\right]^{\frac{1}{2}}\left[\int_{\Omega} \sigma\left|\nabla v_{\omega}-\nabla v_{0}\right|^{2} d \mathbf{r}\right]^{\frac{1}{2}}
\end{aligned}
$$

and so

$$
\int_{\Omega} \sigma\left|\nabla v_{\omega}-\nabla v_{0}\right|^{2} d \mathbf{r} \leq M_{\gamma^{\omega}}^{4} \int_{\Omega} \sigma\left|\nabla v_{\omega}\right|^{2} d \mathbf{r}
$$

Since

$$
\int_{\Omega} \sigma\left|\nabla v_{\omega}\right|^{2} d \mathbf{r} \leq 2 \int_{\Omega} \sigma\left|\nabla v_{\omega}-v_{0}\right|^{2} d \mathbf{r}+2 \int_{\Omega} \sigma\left|\nabla v_{0}\right|^{2} d \mathbf{r},
$$

we obtain the second estimate:

$$
\int_{\Omega} \sigma\left|\nabla v_{\omega}-\nabla v_{0}\right|^{2} d \mathbf{r} \leq \frac{2 M_{\gamma^{\omega}}^{4}}{1-2 M_{\gamma^{\omega}}^{4}} \int_{\Omega} \sigma\left|\nabla v_{0}\right|^{2} d \mathbf{r}
$$

From Theorem 2.1, we can conclude that $v_{\omega}$ and $v_{0}$ is quite close to the real part $v_{\gamma^{\omega}}$ of the complex potential $u_{\gamma^{\omega}}$ at low frequency in similar extent:

$$
v_{\gamma^{\omega}}(\mathbf{r}) \approx v_{\omega}(\mathbf{r}) \approx v_{0}(\mathbf{r}), \quad \mathbf{r} \in \Omega
$$

and hence the well-known elliptic PDE (4) with conductivity coefficient can be replaced by the new alternative elliptic PDE (5) for EIT conductivity imaging. The numerical simulation in Fig. 1 demonstrates our observation (16). The figure shows graphes of $L^{2}$-norms of $v_{\gamma \omega}-v_{0}, v_{\gamma \omega}-v_{\omega}$ and $\sigma$-weighted $L^{2}$ norms of their gradients on the whole unit disk $\Omega$. In the numerical simulation, we used three different anomalies having different admittivity:

$$
\gamma^{\omega}(\mathbf{r})=\sigma(\mathbf{r})+i \omega \epsilon(\mathbf{r})= \begin{cases}\sigma_{b}+i \omega \epsilon_{b}, & \text { in } \Omega \backslash \overline{\left(D_{1} \cup D_{2} \cup D_{3}\right)} \\ \sigma_{c 1}+i \omega \epsilon_{c 1}, & \text { in } D_{1} \\ \sigma_{c 2}+i \omega \epsilon_{c 2}, & \text { in } D_{2} \\ \sigma_{c 3}+i \omega \epsilon_{c 3}, & \text { in } D_{3} .\end{cases}
$$

In the next section, we show that the solution $v_{\omega}$ to $(5)$ has an additional property which the solution $v_{0}$ does not have: Spectroscopic Property. 


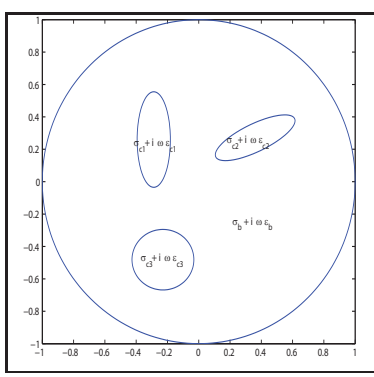

(a)

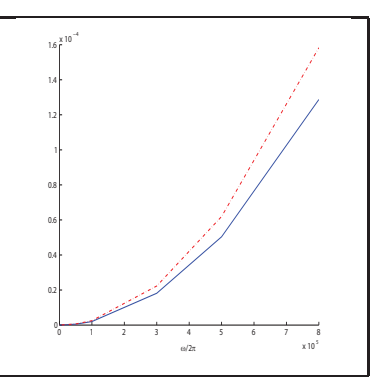

(b)

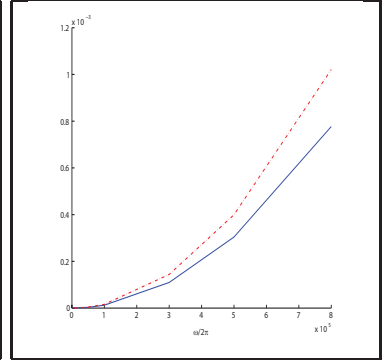

(c)

Figure 1. (a) The tested $2 \mathrm{D}$ disk model having three different inclusions. For the simulation, $\sigma_{b}=1, \sigma_{c 1}=0.3, \sigma_{c 2}=2$, $\sigma_{c 3}=5$ and $\epsilon_{b}=10^{3} \times \epsilon_{0}, \epsilon_{c 1}=5 \times \epsilon_{0}, \epsilon_{c 2}=40 \times \epsilon_{b}$, $\epsilon_{c 3}=5 \times 10^{2} \times \epsilon_{b}$. Here $\epsilon_{0}=8.95 \times 10^{-12}$ is the vacuum permittivity. (b) The solid line is the graph of $\left\|v_{\gamma^{\omega}}-v_{0}\right\|$ and the dashdot line is one of $\left\|v_{\gamma^{\omega}}-v_{\omega}\right\|$ (c) The solid line is the graph of $\left\|\nabla v_{\gamma} \omega-\nabla v_{0}\right\|_{*}$ and the dashdot line is one of $\left\|\nabla v_{\gamma} \omega-\nabla v_{\omega}\right\|_{*}$. Here $\|\cdot\|$ is the traditional $L^{2}$-norm on $\Omega$ and $\|\cdot\|_{*}$ is the weighted $L^{2}$-norm on $\Omega$ defined by $\|\varphi\|_{*}^{2}=$ $\int_{\Omega} \sigma|\varphi|^{2} d \mathbf{r}$.

\section{Spectroscopic property of $v_{\omega}$ in a thin membrane-like anomaly}

In the previous section, we saw that two potentials $v_{\omega}$ and $v_{0}$ are good approximations to the real part $v_{\gamma \omega}$ of the complex potential $u_{\gamma \omega}$ which is the solution to the complex elliptic PDE (2) and they are helpful in EIT conductivity imaging. However there are a remarkable difference between $v_{\omega}$ and $v_{0}$. The difference is that the potential $v_{\omega}$ can show the change of $v_{\gamma^{\omega}}$ with respect to frequency change unlike $v_{0} . v_{0}$ is quite useful in showing the conductivity dependency of $v_{\gamma^{\omega}}$, on the other hand it cannot show the frequency dependency of $v_{\gamma^{\omega}}$, which is a distinct feature of the complex potential $u_{\gamma^{\omega}}$. In this section, we show that the potential $v_{\omega}$ inherits a spectroscopic property from $v_{\gamma}$ using a EIT model containing a thin membrane-like anomaly. See Fig. 2. Here a thin membrane-like anomaly means a thin low-conducting or almost insulating inclusion such as cell membranes and air-filled cracks.

Let the tested specimen occupy a rectangular region $\Omega$ with boundary $\partial \Omega$. Let $\Gamma$ and $\Upsilon$ be portions on the boundary $\partial \Omega$ as in Fig. 2. Let $\Gamma=\left\{\left(x_{1}, \tau\right)|| x_{1} \mid\right.$ $<\ell\}$ and $\Upsilon=\left\{\left(x_{1},-\tau\right)|| x_{1} \mid<\ell\right\}$ for some $\tau>0$. Suppose that a thin membrane-like anomaly $D$ is located inside $\Omega$. We apply a sinusoidal voltage of $V_{0} \sin \omega t$ with the sensor $\Upsilon$ kept at zero potential. Then the resulting time 
harmonic electric potential $u_{\gamma^{\omega}}$ satisfies the following mixed boundary problem:

$$
\left\{\begin{array}{l}
\nabla \cdot\left(\gamma^{\omega}(\mathbf{r}) \nabla u_{\gamma^{\omega}}(\mathbf{r})\right)=0, \quad \mathbf{r} \in \Omega \\
u_{\gamma^{\omega}}(\mathbf{r})=V_{0}, \quad \mathbf{r} \in \Gamma \\
u_{\gamma^{\omega}}(\mathbf{r})=0, \quad \mathbf{r} \in \Upsilon \\
\gamma^{\omega}(\mathbf{r}) \nabla u_{\gamma^{\omega}}(\mathbf{r}) \cdot \mathbf{n}(\mathbf{r})=0, \quad \mathbf{r} \in \partial \Omega \backslash(\Gamma \cup \Upsilon),
\end{array}\right.
$$

where $\mathbf{n}$ is the unit outward normal vector to the boundary $\partial \Omega$. In EIT, from the measurement of the exit current $g_{\gamma^{\omega}}=-(\sigma+i \omega \epsilon) \nabla u_{\gamma^{\omega}} \cdot \mathbf{n}$ on $\Gamma$, we want to reconstruct the thin anomaly $D$.

As mentioned in the previous section, the complex elliptic PDE (17) is hard to handle. So we use the following alternative PDE in order to reconstruct the anomaly $D$ :

$$
\begin{cases}\nabla \cdot\left(\frac{\left|\gamma^{\omega}(\mathbf{r})\right|^{2}}{\sigma} \nabla v_{\omega}(\mathbf{r})\right)=0, & \mathbf{r} \in \Omega \\ v_{\omega}(\mathbf{r})=V_{0}, \quad \mathbf{r} \in \Gamma & \\ v_{\omega}(\mathbf{r})=0, \quad \mathbf{r} \in \Upsilon & \\ \frac{\left|\gamma^{\omega}(\mathbf{r})\right|^{2}}{\sigma} \nabla v_{\omega}(\mathbf{r}) \cdot \mathbf{n}(\mathbf{r})=0, \quad \mathbf{r} \in \partial \Omega \backslash(\Gamma \cup \Upsilon) .\end{cases}
$$

We hope that the above PDE is helpful in determining the anomaly $D$ from the Neumann data $\mathbf{n} \cdot \nabla v_{\omega}$ on $\Gamma$, which may be assumed to be known from the practically measured $\mathbf{n} \cdot \nabla v_{\gamma^{\omega}}$ on $\Gamma$ so that

$$
\mathbf{n} \cdot \nabla v_{\omega}=\mathbf{n} \cdot \nabla v_{\gamma^{\omega}} \quad \text { on } \Gamma \text {. }
$$

Theorem 2.1 convinces us that the pair of $\left.\frac{\partial v_{\omega}}{\partial \mathbf{n}}\right|_{\Gamma}$ and (18) is well substituted for the pair of $\left.\frac{\partial v_{\gamma} \omega}{\partial \mathbf{n}}\right|_{\Gamma}$ and (17) in the sense of inverse problems when the contrast $M_{\gamma^{\omega}}$ is suitably small.

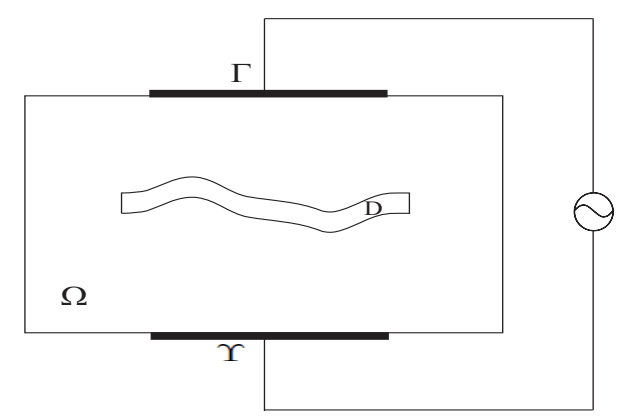

FIGURE 2. Specimen for a thin membrane-like anomaly in EIT. $\Gamma$ and $\Upsilon$ are surface sensors. The exit current $g_{\gamma^{\omega}}=$ $-(\sigma+i \omega \epsilon) \nabla u_{\gamma} \omega \cdot \mathbf{n}$ is measured on $\Gamma$. 


\subsection{Spectroscopic property of $v_{\omega}$}

The notable feature of the alternative potential $v_{\omega}$ is its ability to show the spectroscopic property of the true potential $v_{\gamma^{\omega}}$. So the alternative potential $v_{\omega}$ enables us to have an insight into the behavior of the true potential $v_{\gamma} \omega$, in particular, on the thin membrane-like anomaly. Fig. 3 shows the change of streamlines of $v_{\omega}$ with respect to frequency near a boundary portion of the thin membrane-like anomaly $D$. The simulated specimen $\Omega$ is an open rectangle $(-1,1) \times(-0.5,0.5)$. The surface sensors $\Gamma$ and $\Upsilon$ are located on the boundary portions $\{(x, 0.5) \mid-0.3<x<0.3\}$ and $\{(x,-0.5) \mid-0.3<x<$ $0.3\}$, respectively. The thin membrane-like anomaly $D$ is an open rectangle $(-0.5,0.8) \times(0.08,0.09)$. For simulations, it was supposed that $\sigma_{b}=3$, $\sigma_{c}=2 \times 10^{-4}, \epsilon_{b}=10^{3} \times \epsilon_{0}$, and $\epsilon_{c}=5 \times \epsilon_{b}$. Here $\epsilon_{0}=8.95 \times 10^{-12}$ is the vacuum permittivity. In Fig. 3 streamlines of $v_{\omega}$ on the shaded subregion of the specimen, $(0.3,0.7) \times(0.08,0.12)$, are presented when frequency $\omega / 2 \pi$ is $10 \mathrm{~Hz}, 1 \mathrm{kHz}, 50 \mathrm{kHz}, 100 \mathrm{kHz}, 300 \mathrm{kHz}, 500 \mathrm{kHz}$, and $800 \mathrm{kHz}$. Although it is not carried out because of numerical difficulty, if the thickness of the anomaly is much more thinner than the tested thickness 0.01 , the more intense change of streamlines occurs at a lower frequency range. In EIT, we can detect such change of $v_{\omega}$ with respect to frequency on the surface sensor $\Gamma$ from which geometric information of the anomaly $D$ can be extracted.

\subsection{Spectroscopic change of $\frac{d v_{\omega}}{d \mathbf{n}}$ and anomaly thickness}

The following theorem shows that the frequency differential of the Neumann data $\frac{d v_{\omega}}{d \mathbf{n}}$ on $\Gamma$ is related with the $L^{2}$-norm of the gradient of $v_{\omega}$ on the anomaly D.

Theorem 3.1. For given angular frequency $\omega>0$, the unique solution $v_{\omega}$ to (18) satisfies

$$
\frac{d}{d \omega} \int_{\Gamma} \frac{\partial v_{\omega}}{\partial \mathbf{n}} d s=\alpha \int_{D}\left|\nabla v_{\omega}\right|^{2} d \mathbf{r}
$$

where $\alpha=\frac{2 \omega \sigma_{b}\left(\sigma_{b}^{2} \epsilon_{c}^{2}-\sigma_{c}^{2} \epsilon_{b}^{2}\right)}{V_{0} \sigma_{c}\left|\gamma_{b}^{\omega}\right|^{4}}$.

Proof. For any $h$ such that $\omega+h>0$, let $\omega_{h}=\omega+h$. Let

$$
k=\frac{\sigma_{b}\left|\gamma_{c}^{\omega}\right|^{2}}{\sigma_{c}\left|\gamma_{b}^{\omega}\right|^{2}}-1 \quad \text { and } \quad k_{h}=\frac{\sigma_{b}\left|\gamma_{c}^{\omega_{h}}\right|^{2}}{\sigma_{c}\left|\gamma_{b}^{\omega_{h}}\right|^{2}}-1 .
$$

Note that $v_{\omega}$ and $v_{\omega_{h}}$, respectively, satisfy the following elliptic PDEs:

$$
\nabla \cdot\left(\left(1+k \chi_{D}\right) \nabla v_{\omega}\right)=0 \text { and } \nabla \cdot\left(\left(1+k_{h} \chi_{D}\right) \nabla v_{\omega_{h}}\right)=0 \text { in } \Omega,
$$

where $\chi_{D}$ is the characteristic function on $D$. From (21), we obtain

$$
V_{0} \int_{\Gamma}\left(\frac{\partial v_{\omega_{h}}}{\partial \mathbf{n}}-\frac{\partial v_{\omega}}{\partial \mathbf{n}}\right) d s=\left(k_{h}-k\right) \int_{D}\left(\nabla v_{\omega} \cdot \nabla v_{\omega_{h}}\right) d \mathbf{r} .
$$




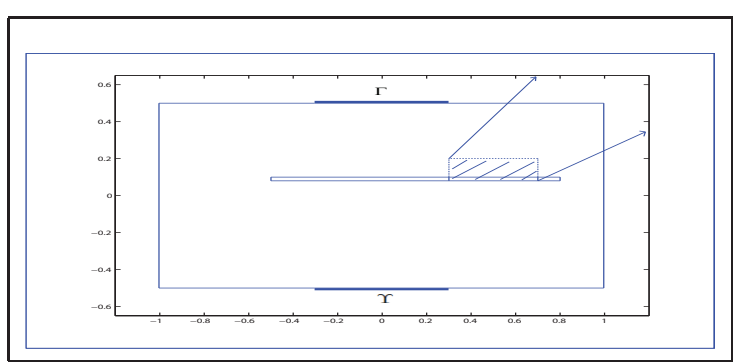

(a)

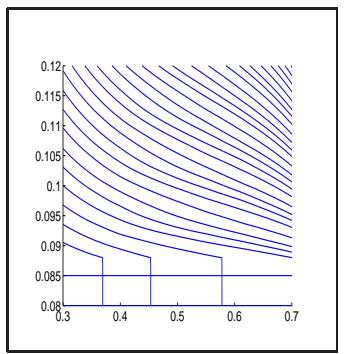

(c)

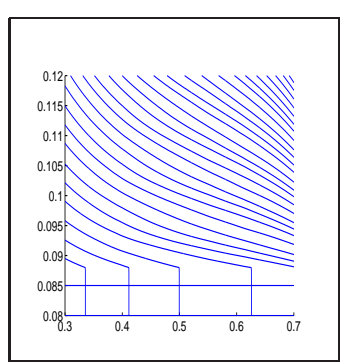

(f)

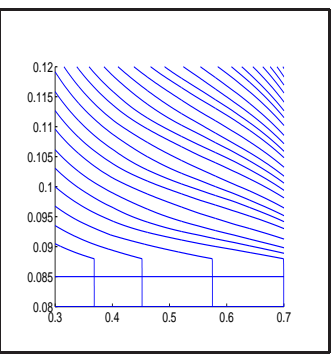

(d)

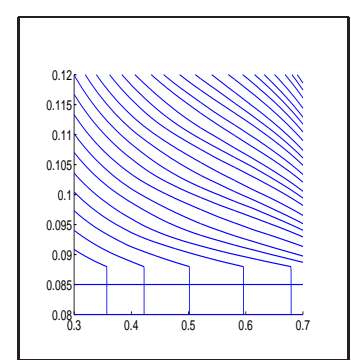

$(\mathrm{g})$

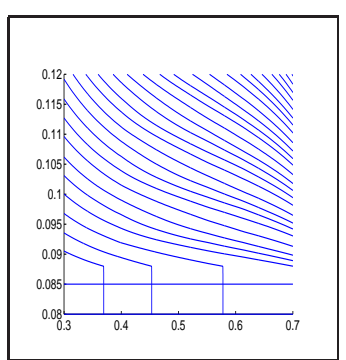

(b)

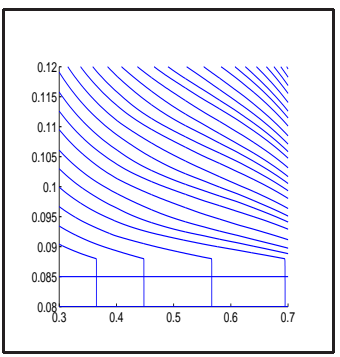

(e)

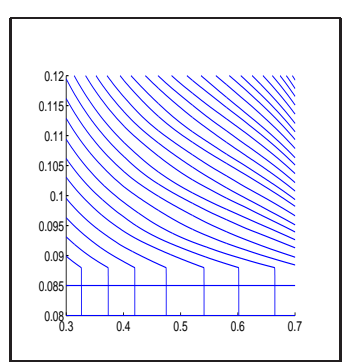

(h)

FiguRE 3. (a) The tested model. The streamlines of the alternative potential $v_{\omega}$ on the shaded subregion are presented when (b) $\omega / 2 \pi=10 \mathrm{~Hz}$, (c) $1 \mathrm{kHz}$, (d) $50 \mathrm{kHz}$, (e) $100 \mathrm{~Hz}$, (f) $300 \mathrm{kHz},(\mathrm{g}) 500 \mathrm{kHz}$, and (h) $800 \mathrm{kHz}$.

Since $k_{h}-k=\frac{\sigma_{b} h(2 \omega+h)\left(\sigma_{b}^{2} \epsilon_{c}^{2}-\sigma_{c}^{2} \epsilon_{b}^{2}\right)}{\sigma_{c}\left|\gamma_{b}^{\omega}\right|^{2}\left|\gamma_{b}^{\omega h}\right|^{2}}$, we have

$$
\frac{1}{h} \int_{\Gamma}\left(\frac{\partial v_{\omega_{h}}}{\partial \mathbf{n}}-\frac{\partial v_{\omega}}{\partial \mathbf{n}}\right) d s=\frac{\sigma_{b}(2 \omega+h)\left(\sigma_{b}^{2} \epsilon_{c}^{2}-\sigma_{c}^{2} \epsilon_{b}^{2}\right)}{V_{0} \sigma_{c}\left|\gamma_{b}^{\omega}\right|^{2}\left|\gamma_{b}^{\omega_{h}}\right|^{2}} \int_{D}\left(\nabla v_{\omega} \cdot \nabla v_{\omega_{h}}\right) d \mathbf{r}
$$


and taking the limit as $h \rightarrow 0$, we have

$$
\frac{d}{d \omega} \int_{\Gamma} \frac{\partial v_{\omega}}{\partial \mathbf{n}} d s=\frac{2 \omega \sigma_{b}\left(\sigma_{b}^{2} \epsilon_{c}^{2}-\sigma_{c}^{2} \epsilon_{b}^{2}\right)}{V_{0} \sigma_{c}\left|\gamma_{b}^{\omega}\right|^{4}} \int_{D}\left|\nabla v_{\omega}\right|^{2} d \mathbf{r} .
$$

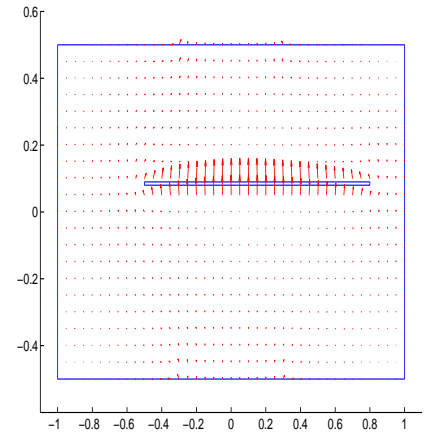

(a)

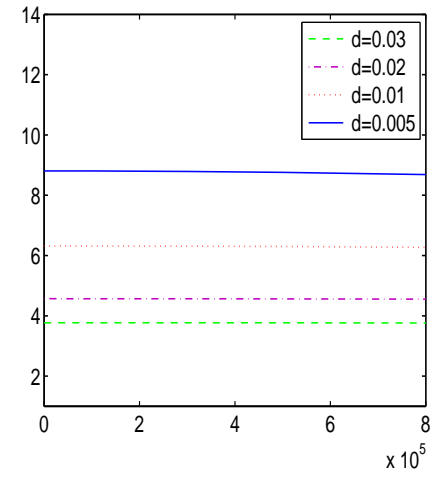

(b)

Figure 4. (a) Plot of the gradient vector $\nabla v_{\omega}$. (b) The graph of $\frac{1}{\alpha} \frac{d}{d \omega} \int_{\Gamma} \frac{\partial v_{\omega}}{\partial \mathbf{n}} d s$. The horizontal axis is for frequency $\omega / 2 \pi$. The tested anomaly $D$ is an open rectangle $(-0.5,0.8) \times(0.08,0.08+d)$ for $d=0.005,0.01,0.02$, and 0.03. The numerical result shows that $\frac{d}{d \omega} \int_{\Gamma} \frac{\partial v_{\omega}}{\partial \mathbf{n}} d s \propto \frac{1}{d}$.

The frequency dependent behavior of $v_{\omega}$, for instance shown in Figure 3, is embedded in the Neumann data $\left.\frac{\partial v_{\omega}}{\partial \mathbf{n}}\right|_{\Gamma}$ and the extent of its changes depends on the admittivity of the object $\Omega$ and geometric features of the anomaly. In specific case where the anomaly $D$ is a thin low-conducting material like crack or membrane located between two surface sensors as shown in Figure 2, the anomaly thickness can be well reflected in this frequency dependent behavior of $v_{\omega}$ more than any other factor. Returning to Theorem 3.1, the right hand side of (20) can be understood in terms of the anomaly thickness. Roughly speaking, we expect that

$$
\int_{D}\left|\nabla v_{\omega}\right|^{2} d \mathbf{r} \propto \frac{V_{0}^{2}}{d}
$$

This expectation is based on a physical law about a dielectric capacitor.

First we consider the zero frequency case. If all widths of the tested specimen, the thin rectangular anomaly $D$, and two surface sensors are same, for example $\ell$, then we have

$$
\int_{D}\left|\nabla v_{0}\right|^{2} d \mathbf{r}=\frac{V_{0}^{2} \ell}{d}
$$


Even though otherwise they do not have the same width, if the width of the thin anomaly $D$ is bigger than one of surface sensors, it is expected that $\int_{D}\left|\nabla v_{0}\right|^{2} d \mathbf{r} \propto \frac{V_{0}^{2}}{d}$. Because $v_{0} \approx V_{0}$ near the center of the upper side of the thin anomaly $D$ and $v_{0} \approx 0$ near the center of the bottom side of $D$. (23) can be followed from Theorem 2.1.

From Theorem 3.1 it follows that the rate of change of the measured Neumann boundary data $\frac{d v_{\omega}}{d \mathbf{n}}$ on $\Gamma$ with respect to angular frequency $\omega$ reflects the square of the $L^{2}$-norm of the gradient $\nabla v_{\omega}$ only on the anomaly $D$, and hence we can conclude that the frequency differential data $\frac{d}{d \omega} \int_{\Gamma} \frac{\partial v_{\omega}}{\partial \mathbf{n}} d s$ has an inverse proportion to the thickness od the anomaly $D$ :

$$
\frac{d}{d \omega} \int_{\Gamma} \frac{\partial v_{\omega}}{\partial \mathbf{n}} d s \propto \text { Reciprocal of thickness of the anomaly } D \text {. }
$$

The numerical results in Fig. 4(b) demonstrates our observation.

\section{Conclusion}

In this paper, the usefulness of the potential $v_{\omega}$, which is the unique solution to $(5)$, was considered. Since $v_{\omega}$ is close to the traditional solution $v_{0}$ to the standard elliptic PDE with the conductivity coefficient, it can be used for conductivity imaging in EIT. Also, using the alternative potential $v_{\omega}$, we can observe the spectroscopic property of the true potential $v_{\gamma^{\omega}}$ with respect to frequency $\frac{\omega}{2 \pi}$ without difficulty to deal with the coupled system (6). In particular, Theorem 3.1 implies that such spectroscopic change in the Neumann data $\frac{d v_{\omega}}{d \mathbf{n}}$ on the boundary is closely related with the total amount of $\left|\nabla v_{\omega}\right|^{2}$ on the anomaly $D$. If the anomaly $D$ is a thin low-conducting material such as air-filled cracks or tissue membranes, this spectroscopic change in the Neumann data $\frac{d v_{\omega}}{d \mathbf{n}}$ on the boundary is inversely proportional to the thickness of the anomaly $D$, and the alternative potential $v_{\omega}$ can be used in detecting the thickness of the thin membrane-like anomaly. Even considering this, the alternative potential $v_{\omega}$ deserves many attentions in EIT as much as $v_{0}$.

The potential $v_{\omega}$ can not only replace two potentials $v_{0}$ and $v_{\gamma \omega}$ as mentioned in previous sections, but also enable us to analysis the interrelation between the real part an imaginary parts of the true complex potential $u_{\gamma^{\omega}}$, which is indispensable for understanding the complex PDE (2) and its applications. In the next work, the interrelation between the real part and imaginary parts of the true complex potential $u_{\gamma^{\omega}}$ will be investigated using the alternative potential $v_{\omega}$.

\section{References}

[1] H. Ammari, E. Beretta, and E. Francini, Reconstruction of thin conductivity imperfections, Appl. Anal. 83 (2004), no. 1, 63-76.

[2] H. Ammari, O. Kwon, J. K. Seo, and E. J. Woo, T-Scan electrical impedance imaging system for anomaly detection, SIAM J. Appl. Math. 65 (2004), no. 1, 252-266. 
[3] H. Ammari and J. K. Seo, An accurate formula for the reconstruction of conductivity Inhomogeneity, Adv. in Appl. Math. 30 (2003), no. 4, 679-705.

[4] E. Beretta, E. Francini, and M. Vogelius, Asymptotic formulas for steady state voltage potentials in the presence of thin inhomogeneities. A rigorous error analysis, J. Math. Pures Appl. (9) 82 (2003), no. 10, 1277-1301.

[5] M. Brühl and M. Hanke, Numerical implementation of two noniterative methods for locating inclusions by impedance tomography, Inverse Problems 16 (2000), no. 4, 10291042.

[6] M. Cheney, D. Isaacson, and J. C. Newell, Electrical impedance tomography, SIAM Rev. 41 (1999), no. 1, 85-101.

[7] S. Gabriel, R. W. Lau, and C. Gabriel, The dielectric properties of biological tissues II. measurements in the frequency range $10 \mathrm{~Hz}$ to $20 \mathrm{GHz}$, Phys. Med. Biol. 41 (1996), $2251-2269$.

[8] H. Griffiths, H. T. L. Leung, and R. J. Williams, Imaging the complex impedance of the thorax, Clin. Phys. Physiol. Meas. 13 A (1992), 77-81.

[9] S. Grimnes and O. G. Martinsen, Bioimpedance and Bioelectricity Basics, Oxford, UK: Elsevier, 2008.

[10] J. Gu and L. Y. Yu, Influence of geometric factors on crack depth measurement using the potential drop technique, NDT Int. 23 (1990), 161-164.

[11] D. Holder, Electrical Impedance Tomography: Methods, History and Applications, Bristol, UK: IOP Publishing, 2005.

[12] H. Jain, D. Isaacson, P. M. Edic, and J. C. Newell, Electrical impedance tomography of complex conductivity distributions with noncircular boundary, IEEE Trans. Biomed. Eng. 44 (1997), 1051-1060.

[13] S. Kim, M. K. Choi, E. J. Lee, J. K. Seo, and E. J. Woo, Spectroscopic admittivity imaging of membrane structure, to appear.

[14] S. Kim, J. Lee, J. K. Seo, E. J. Woo, and H. Zribi, Multifrequency trans-admittance scanner: mathematical framework and feasibility, SIAM J. Appl. Math. 69 (2008), no. $1,22-36$.

[15] S. Kim, J. K. Seo, and T. Ha, A nondestructive evaluation method for concrete voids: frequency differential electrical impedance scanning, SIAM J. Appl. Math. 69 (2009), no. $6,1759-1771$.

[16] H. Lee and W. K Park, Location search algorithm of thin conductivity inclusions via boundary measurements, Mathematical methods for imaging and inverse problems, 21729, ESAIM Proc., 26, EDP Sci., Les Ulis, 2009.

[17] T. I. Oh, J. Lee, J. K. Seo, S. W. Kim, and E. J. Woo, Feasibility of breast cancer lesion detection using multi-frequency trans-admittance scanner $(T A S)$ with $10 \mathrm{~Hz}$ to $500 \mathrm{kHz}$ bandwidth, Physiol. Meas. 28 (2007), 71-84.

[18] W. K. Park and D. Lesselier, Reconstruction of thin electromagnetic inclusions by a level-set method, Inverse Problems 25 (2009), no. 8, 1-24.

[19] J. K. Seo, O. Kwon, H. Ammari, and E. J. Woo, Mathematical framework and anomaly estimation algorithm for breast cancer detection: electrical impedance technique using TS2000 configuration, IEEE Trans. Biomed. Eng. 51 (2004), no. 11, 1898-1906.

[20] E. Somersalo, M. Cheney, and D. Isaacson, Existence and uniqueness for electrode models for electric current computed tomography, SIAM J. Appl. Math. 52 (1992), no. 4, 1023-1040.

Division of LiBERAL ARTS

HANBAT NATIONAL UNIVERSITY

DAEJEON 305-719, KOREA

E-mail address: sungwhan@hanbat.ac.kr 